\title{
Calibration transfer for bioprocess Raman monitoring using Kennard Stone Piecewise Direct Standardization and multivariate algorithms
}

\author{
Laure Pétillot ${ }^{1}$, Fiona Pewny ${ }^{2}$, Martin Wolf ${ }^{2}$, Célia Sanchez ${ }^{1}$, Fabrice Thomas ${ }^{1}$, Johan \\ Sarrazin $^{1}$, Katharina Fauland ${ }^{2}$, Herman Katinger ${ }^{2}$, Charlotte Javalet $^{1}$, and Christophe \\ Bonneville ${ }^{1}$
}

${ }^{1}$ RESOLUTION Spectra Systems

${ }^{2}$ Polymun Scientific

May 5, 2020

\begin{abstract}
In the biopharmaceutical industry, Raman spectroscopy is now a proven PAT tool that enables in-line simultaneous monitoring of several CPPs and CQAs in real-time. However, as Raman monitoring requires multivariate modeling, variabilities unknown by models can impact the monitoring prediction accuracy. With the widespread use of Raman PAT tools, it is necessary to fix instrumental variability impacts, encountered for instance during a device replacement. In this work, we investigated the impact of instrumental variability between probes inside a multi-channel analyzer and between two analyzers, and explored solutions to correct them on model prediction errors in cell cultures. We found that the Kennard Stone Piecewise Direct Standardization (KS PDS) method enables to lower model prediction errors and that only one batch with the unknown device in the calibration dataset was sufficient to correct the prediction gap induced by instrumental variability. As a matter of fact, during device replacement a first cell culture monitoring can be performed with the KS PDS method. Then, the new data obtained can be inserted in the calibration dataset to integrate instrumental variability in the chemometric model. This methodology provides good multivariate calibration model prediction errors throughout the instrumental changes.
\end{abstract}

\section{Hosted file}

Petillot et al. Final.pdf available at https://authorea.com/users/293945/articles/422087calibration-transfer-for-bioprocess-raman-monitoring-using-kennard-stone-piecewisedirect-standardization-and-multivariate-algorithms 